Internist 2021 - 62:326-332

https://doi.org/10.1007/s00108-021-00959-5

Angenommen: 20. Januar 2021

Online publiziert: 13. Februar 2021

๑) Springer Medizin Verlag GmbH, ein Teil von Springer Nature 2021

\section{Redaktion}

M. Wehling, Mannheim

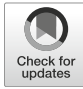

\section{Klimek ${ }^{1} \cdot$ J. Eckrich ${ }^{2} \cdot$ J. Hagemann ${ }^{2} \cdot$ I. Casper ${ }^{1} \cdot$ J. Huppertz ${ }^{2}$}

'Zentrum für Rhinologie und Allergologie, Wiesbaden, Deutschland

${ }^{2}$ Universitätsklinik für Hals-Nasen-Ohrenheilkunde und Kopf-Hals-Chirurgie, Universitätsmedizin der Johannes Gutenberg-Universität Mainz, Mainz, Deutschland

\title{
Allergische Reaktionen auf COVID-19-Impfstoffe - Evidenz und praxisorientiertes Vorgehen
}

\section{Hintergrund}

Zur vorbeugenden Behandlung der „coronavirus disease 2019“ (COVID-19) wurden in einer beispiellosen weltweiten Forschungsanstrengung Sicherheit und Wirksamkeit neuer Impfstoffplattformen untersucht, die noch nie zuvor am Menschen eingesetzt wurden. Weniger als ein Jahr nach der Entdeckung der Severe-acute-respiratorysyndrome-coronavirus-2(SARS-CoV-

2)-Virussequenz wurden Impfstoffe in zahlreichen Ländern für den Routineeinsatz zugelassen und es wurde mit Massenimpfungen begonnen. Die bislang in der Europäischen Union (EU) zugelassenen mRNA-Impfstoffe gegen SARS-CoV-2 BNT162b2 (BioNTech/ Pfizer) und mRNA-1273 (Moderna) basieren auf einer ähnlichen lipidbasierten Nanopartikelträgertechnologie; die Lipidkomponenten unterscheiden sich jedoch. Allergische Reaktionen und Anaphylaxien machen einen wesentlichen Teil der bislang bekannt gewordenen unerwünschten Reaktionen auf diese Impfstoffe aus. Insgesamt sind sie aber dennoch selten.

In den klinischen Studien der Phase III wurden keine ernsthaften Nebenwirkungen festgestellt [1]. Leichte lokale Nebenwirkungen wie Schmerzen, Rötungen und Schwellungen an der Einstichstelle wurden bei den Impfstoffen häufiger beobachtet als bei Placebo. Systemische Symptome wie Fieber, Müdigkeit, Kopfschmerzen sowie Muskelund Gelenkschmerzen wurden ebenfalls etwas häufiger unter den Impfstoffen als unter Placebo beobachtet, und die meisten Symptome traten in den ersten 24-48 h nach der Impfung auf [1]. In den klinischen Studien der Phase I-III zu den mRNA-Impfstoffen BNT162b2 und mRNA-1273 wurden potenzielle Teilnehmer mit Vorgeschichte einer allergischen Reaktion auf einen Bestandteil des Impfstoffs ausgeschlossen, bei BNT162b2 zusätzlich auch Teilnehmer mit einer schweren Allergie in der Vorgeschichte in Verbindung mit irgendeinem Impfstoff [1, 2] Unerwünschte Ereignisse als allergische Reaktionen traten in beiden Studien in der Placebo- (Kochsalzlösung) und der Impfstoffgruppe gleichermaßen auf [1].

Die britische Arzneimittelbehörde MHRA (Medicines \& Healthcare Products Regulatory Agency) hat als erste eine Notfallzulassung für den mRNAImpfstoff von BioNTech/Pfizer erteilt. Am 08.12.2020, innerhalb eines Tages nach Beginn des britischen Massenimpfprogramms für Mitarbeiter des Gesundheitswesens und ältere Erwachsene, wurden Anaphylaxien bei 2 Frauen im Alter von 40 und 49 Jahren mit vorbekannten Nahrungsmittel- und Medikamentenallergien beobachtet. Ähnliches wurde auch aus den USA berichtet, wo die Food and Drug Administration (FDA) am 11.12.2020 eine Notfallzulassung („emergency use authorization“ [EUA]) für BNT162b2 erteilte. In der Folge traten anaphylaktische Reaktionen auch in den USA und anderen Ländern auf $[3,4]$.

Als Reaktion auf die Anaphylaxiefälle in Großbritannien hat die MHRA ent- schieden, dass Personen, bei denen in der Vergangenheit eine anaphylaktische Reaktion auf ein Lebensmittel, ein Medikament oder einen Impfstoff aufgetreten ist, vorerst nicht mit BNT162b2 geimpft werden sollten. In den USA wurde für beide mRNA-Impfstoffe empfohlen, alle Personen auszuschließen, bei denen in der Vorgeschichte eine schwere oder sofortige (innerhalb von $4 \mathrm{~h}$ ) allergische Reaktion im Zusammenhang mit einem der Bestandteile des Impfstoffs, einschließlich von Polyethylenglykol (PEG) und PEGDerivaten wie Polysorbat, aufgetreten ist [5].

Ende Dezember 2020 waren in den USA etwa 2 Mio. Menschen geimpft (28. Dezember, 2020). Die Inzidenz von Anaphylaxien im Kontext der SARSCoV-2-Impfung mit den mRNA-Impfstoffen scheint etwa zehnmal so hoch zu sein wie die Inzidenz, die mit früheren Impfstoffen beobachtet wurde, nämlich etwa 1 auf 100.000 Impfinjektionen im Vergleich zu $1 \mathrm{zu} 1.000 .000$ bei anderen Impfungen.

\section{Mögliche anaphylaxie- auslösende Inhaltsstoffe in den mRNA-COVID-19-Impfstoffen}

Die aktuell in westlichen Industrieländern kommerziell verfügbaren COVID19-Impfstoffe BNT162b2 und mRNA1273 enthalten keine der „klassischen“ allergieinduzierenden Komponenten wie Gelatine, Ovalbumin oder andere Hühnereiproteine, Kuhmilchproteine (hauptsächlich verantwortlich für Soforttypreaktionen), Thiomersal, Alumi- 


\begin{tabular}{|c|c|}
\hline BNT162b2 & mRNA-1273 \\
\hline $\begin{array}{l}\text { Nukleosidmodifizierte mRNA, die für das virale } \\
\text { Spike(S)-Glykoprotein von SARS-CoV-2 codiert }\end{array}$ & $\begin{array}{l}\text { Nukleosidmodifizierte mRNA, die für das virale } \\
\text { Spike(S)-Glykoprotein von SARS-CoV-2 codiert }\end{array}$ \\
\hline $\begin{array}{l}\text { 2-(Polyethylenglykol-2000)-N,N-ditetrade- } \\
\text { cylacetamid }\end{array}$ & $\begin{array}{l}\text { Polyethylenglykol-2000-dimyristoyl-glycerol } \\
\text { (DMG) }\end{array}$ \\
\hline 1,2-Distearoyl-sn-glycero-3-phosphocholin & 1,2-Distearoyl-sn-glycero-3-phosphocholin \\
\hline Cholesterin & Cholesterin \\
\hline $\begin{array}{l}\text { [(4-Hydroxybutyl)azandiyl]bis(hexan-6,1-diyl)- } \\
\text { bis(2-hexyldecanoat) }\end{array}$ & SM-102 (Patent von Moderna) \\
\hline Kaliumchlorid & Tromethamin \\
\hline Monobasisches Kaliumphosphat & Tromethaminhydrochlorid \\
\hline Natriumchlorid & Essigsäure \\
\hline Dibasisches Natriumphosphatdihydrat & Natriumacetat \\
\hline Saccharose & Saccharose \\
\hline \multicolumn{2}{|c|}{ SARS-CoV-2 "severe acute respiratory syndrome coronavirus 2 " } \\
\hline
\end{tabular}

nium, Phenoxyethanol oder Formaldehyd (hauptsächlich verantwortlich für Spättypreaktionen). Reste antimikrobieller Substanzen wie Neomycin oder Substanzen wie Latex, Hefen und Dextran sind ebenfalls nicht enthalten und auch Konservierungsmittel oder sonstige Zusatzstoffe werden nicht benötigt $[1,2,4]$.

Daher ist zunächst zu klären, welche Bestandteile von BNT162b2 und mRNA1273 (• Tab. 1) generell Anaphylaxien auslösen können.

Beide Impfstoffe bestehen aus nukleosidmodifizierter mRNA, die für das virale Spike(S)-Glykoprotein von SARSCoV-2 codiert. Diese SARS-CoV-2Virus-mRNA ist in Lipidnanopartikel (LNP) eingewickelt, die helfen, die mRNA in die humanen Zellen zu transportieren. Die liposomale Hülle besteht im Wesentlichen aus Phospholipiden (häufig modifiziert durch inkorporierte Cholesterine), die den im wässrigen Milieu befindlichen RNA-Impfstoff umschließen [6].

Diese als Nanopartikel bezeichnete Formulierung, die sowohl als Träger als auch als Stabilisator der RNA dient, ist mit zusätzlichen Hilfsstoffen versetzt, wie dem Polymer PEG, das unter anderem eine sterische Barriere gegen vorschnellen Abbau der Liposomen durch das retikuloendotheliale System bildet [7].

Die LNP wirken zusätzlich auch als immunverstärkendes Adjuvans. Sie sind „pegyliert", das heißt, chemisch an PEGMoleküle gebunden, die die Außenseite der Partikel bedecken und deren Stabilität und Lebensdauer erhöhen.

\section{》) Liposomen können in Abhängigkeit von Größe und Zusammensetzung das Immunsystem aktivieren}

Prinzipiell können diese Komponenten einzeln oder in Kombination als Induktoren von Anaphylaxien fungieren. So kann sowohl einzel- als auch doppelsträngige RNA das angeborene Immunsystem stimulieren, beispielsweise über die Tolllike-Rezeptoren TLR3 und TLR7/8, und zur exzessiven Freisetzung unterschiedlicher immunaktivierender Zytokine führen. Des Weiteren können Liposomen in Abhängigkeit von Größe, Zusammensetzung, Aufbau und Oberflächenladung das Immunsystem aktivieren, und die Bildung von Antikörpern gegen spezifische Bestandteile induzieren. Sodann können auch Hilfsstoffe Anaphylaxien auslösen. Eine besondere Rolle könnte in diesem Zusammenhang PEG spielen [3, 4].

Polyethylenglykol. PEG ist in beiden Impfstoffen enthalten. Es ist beschrieben, dass anaphylaktische Reaktionen durch PEG ausgelöst werden können [8-12].

PEG wurde noch nie in einem zugelassenen Impfstoff verwendet, aber es ist in vielen Medikamenten und Alltagsprodukten enthalten. Patienten, die zuvor mit PEG in Berührung gekommen sind, können hohe Mengen an Antikörpern gegen PEG aufweisen, was sie dem Risiko einer anaphylaktischen Reaktion auf den Impfstoff aussetzen könnte [8].

PEG werden in zahlreichen alltäglichen Produkten wie Zahnpasta, Zahnseide, Shampoos, Kosmetikprodukten, Vitaminpräparaten und Lutschbonbons als Verdickungs- oder Lösungsmittel, Weichmacher oder Feuchtigkeitsträger verwendet, und sie werden seit Jahrzehnten als Abführmittel (Macrogol) eingesetzt. Des Weiteren kommen sie in einer Vielzahl an Arzneimitteln vor, so etwa in Antibiotika, Analgetika, Antiemetika, Antiepileptika, Antidepressiva, Antikoagulanzien, ja selbst in antiallergischen Medikamenten wie Kortikosteroidpräparaten und Antihistaminika, ebenso in medizinisch eingesetzten Produkten wie Desinfektionsmitteln oder Ultraschallgelen. Auch eine zunehmende Zahl von Biopharmazeutika und Biologika enthält pegylierte Verbindungen $[13,14]$.

PEG sind hydrophile Polyetherverbindungen, die zahlreiche Synonyme haben, beispielsweise Macrogol. Das Molekulargewicht der verschiedenen PEG reicht von 300 bis $35.000 \mathrm{~g} / \mathrm{mol}$ und Überempfindlichkeitsreaktionen können durch PEG aller Molekulargewichte ausgelöst werden, wobei sowohl Sofortals auch Spättypreaktionen beschrieben wurden $[11,12]$.

\section{》) Allergische Reaktionen können durch PEG-spezifische Immunglobuline ausgelöst werden}

Allergische Reaktionen können durch PEG-spezifische Immunglobulin-E(IgE)-, aber auch durch Immunglobulin-M(IgM)/ Immunglobulin-G(IgG)-Antikörper ausgelöst werden. $\mathrm{Zu}$ anaphylaktischen Reaktionen kann es auch im Rahmen einer komplementaktivierungsbedingten Pseudoallergie (CARPA) kommen, die vor allem durch Medikamente auf Nanopartikelbasis ausgelöst wird, welche oft pegyliert sind [15].

Unabhängig von der Pegylierung haben Liposomen das Potenzial, Komplement unspezifisch zu aktivieren, abhän- 
gig von ihrer unterschiedlichen Oberflächenstruktur und Ladung. Wichtige Mediatoren sind hier die Komplementprodukte C3a, C4a und C5a (Anaphylatoxine; [8]).

Tromethamin/Trometamol. Zusätzlich und im Gegensatz zum Impfstoff BNT162b2 enthält mRNA-1273 Tromethamin, auch Trometamol genannt (Summenformel: $\mathrm{C}_{4} \mathrm{H}_{11} \mathrm{NO}_{3}$; [3]).

Tromethamin/Trometamol ist ein organisches Amin, das in verschiedenen Medikamenten zur topischen, enteralen oder parenteralen Verabreichung und auch in kosmetischen Produkten als Emulgator verwendet wird. Es wurden Kontaktsensibilisierungen und Allergien gegen Tromethamin/Trometamol beschrieben und auch Anaphylaxien bei Verwendung als Hilfsstoff in iodierten Röntgenkontrastmitteln und Kontrastmitteln auf Gadoliniumbasis [3].

Weitere immunologische Effekte der Impfstoffe sind derzeit nicht zu erkennen. Dass die im mRNA-Impfstoff verwendete Erbinformation des Virus dauerhaft in das menschliche Genom integriert werden könnte, erscheint weitgehend ausgeschlossen. Bislang gibt es keine Hinweise, dass die von den humanen Zellen nach der Impfung aufgenommene mRNA in DNA umgeschrieben werden könnte. Die nach intramuskulärer Injektion in das Zytoplasma von Muskel- und Immunzellen aufgenommene einsträngige mRNA müsste dafür in den Zellkern gelangen und darüber hinaus in DNA umgeschrieben werden, was allein aufgrund fehlender Enzymausstattung in humanen Zellen nicht möglich ist.

Vielmehr besteht die Problematik von mRNA-Impfungen eher in einer zu kurzen Wirkdauer der mRNA bei möglichem extrazellulärem Abbau und in einerfehlenden Aufnahme in die menschlichen Zellen. Daher sind aufwendige Verfahren notwendig, die die mRNA durch Einschluss in LNP vor zu rascher Degradierung schützen. $\mathrm{Ob}$ diese nun für die Auslösung der beobachteten Anaphylaxien verantwortlich sind, ist nicht abschließend geklärt.

Internist 2021 · 62:326-332 https://doi.org/10.1007/s00108-021-00959-5

(c) Springer Medizin Verlag GmbH, ein Teil von Springer Nature 2021

L. Klimek · J. Eckrich · J. Hagemann · I. Casper · J. Huppertz

\section{Allergische Reaktionen auf COVID-19-Impfstoffe - Evidenz und praxisorientiertes Vorgehen}

\section{Zusammenfassung}

Weniger als ein Jahr nach der Entdeckung des "severe acute respiratory syndrome coronavirus 2" (SARS-CoV-2) wurden in zahlreichen Ländern Impfstoffe für den Routineeinsatz zugelassen und bereits in der Massenimpfung eingesetzt, darunter die mRNA-Impfstoffe BNT162b2 und mRNA-1273. Allergische Reaktionen und Anaphylaxien machen einen wesentlichen Teil der bislang beobachteten unerwünschten Reaktionen auf diese Impfstoffe aus, sind insgesamt aber selten. Die Inzidenz von Anaphylaxien im Kontext der SARS-CoV-2-Impfung mit den mRNA-Impfstoffen scheint mit ungefähr 1 auf 100.000 Impfinjektionen etwa zehnmal so hoch zu sein wie bei früheren Impfstoffen. Ein Schwerpunkt des vorliegenden Beitrags ist die systematische Betrachtung der Inhaltsstoffe von mRNA-Impfstoffen gegen die "Coronavirus disease 2019" (COVID19). Dabei wird auf die Unterschiede zu etablierten Impfstoffen eingegangen und das allergische Potenzial von Liposomen,
Polyethylenglykol, Tromethamin/Trometamol sowie mRNA diskutiert. Ein weiterer Fokus sind die klinische Präsentation und der Verlauf allergischer Reaktionen nach Applikation der COVID-19-Impfstoffe. Im Anschluss wird auf das therapeutische Vorgehen bei anaphylaktischer Reaktion und die dafür notwendigen Medikamente und medizinischen Materialien eingegangen. $\mathrm{Zu}$ beachten ist, dass eine Anaphylaxie jeden Geimpften treffen kann, unabhängig davon, ob bereits allergische Erkrankungen vorbekannt sind. Daher muss jede Impfstelle und jeder Impfarzt darauf vorbereitet sein, schwere allergische Reaktionen zu erkennen und zu behandeln.

\section{Schlüsselwörter}

Unerwünschte Wirkungen und Reaktionen auf Arzneimittel - Massenimpfung/SARSCoV-2 - Anaphylaktische Reaktion . Liposomen · Polyethylenglykol

\section{Allergic reactions to COVID-19 vaccines: evidence and practice- oriented approach}

\section{Abstract}

Less than a year after the first detection of severe acute respiratory syndrome coronavirus 2 (SARS-CoV-2), vaccines have been approved for routine use in numerous countries and have already been used in mass vaccination programs. Vaccines include the mRNA BNT162b2 and mRNA 1273. Allergic reactions and anaphylaxis account for a substantial proportion of the adverse reactions to these vaccines observed to date, but overall they are rare. The incidence of anaphylaxis in the context of SARS-CoV-2 vaccination with the mRNA vaccines appears to be approximately 10-fold higher than with previous vaccines, at approximately 1 per 100,000 vaccine injections. One focus of the present article is a systematic review of the components of mRNA vaccines against " coronavirus disease 2019" (COVID-19). Differences from established vaccines are addressed and the allergic potential of liposomes, polyethylene glycol, tromethamine/trometamol, and mRNA are discussed. Another focus is on the clinical presentation and course of allergic reactions to the COVID-19 vaccines. This is followed by a discussion of the therapeutic approach to anaphylactic reactions, as well as the drugs and medical supplies required to treat them. It is important to note that any vaccinee may be affected by anaphylaxis, regardless of whether or not allergic diseases are already known. Therefore, every vaccination site and every vaccinator must be prepared to recognize and treat severe allergic reactions.

Keywords

Drug-related side effects and adverse reactions - Mass vaccination/SARS-CoV- 2 . Anaphylaxis - Liposomes · Polyethylene glycols 


\section{Erkennen einer allergischen Reaktion auf COVID-19- Impfstoffe}

Allergische Reaktionen auf eine COVID19-Impfung können im Sinne einer anaphylaktischen Reaktion mit Symptomen an Haut, Atemwegen, Gastrointestinaltrakt und kardiovaskulärem System auftreten. Die anaphylaktische Reaktion wird in vier Schweregrade unterteilt [16, 17].

Die Reaktion kann mit den Prodromalbeschwerden

- Juckreiz,

- Brennen im Bereich der Handinnenflächen, Fußsohlen und im Genitalbereich,

- metallischem Geschmack,

- Angst,

- Kopfschmerzen und

- Desorientierung

beginnen. Häufig kommt es dann zu

- Urtikaria,

- Mundschleimhautbeschwerden,

- Schluckbeschwerden und auch

- Schwellungen im Rachenbereich und Bronchialkonstriktion mit Dyspnoe.

Bei schwerem Verlauf können insbesondere die Atemwegsobstruktion und eine kardiovaskuläre Mitbeteiligung zu einem letalen Verlauf führen.

\section{》) Das frühe Auftreten der Symptomatik macht eine schwere Reaktion wahrscheinlicher}

Charakteristischerweise beginnt die Symptomatik plötzlich und kurz nach der Applikation eines Allergens, das frühe Auftreten macht eine schwere Reaktion wahrscheinlicher als ein verzögertes Auftreten der Reaktion [16].

Beim Impfstoff BNT162B2 traten drei Viertel aller allergischen Reaktionen innerhalb von 15 min nach der Impfung auf [18]. Die differenzialdiagnostische Abgrenzung zu Angst/Panikreaktionen mit Hyperventilation kann schwierig sein. Wichtig sind die Einordnung von Symptomen und bei Verdacht auf Anaphylaxie die unmittelbare Einleitung einer adäquaten Behandlung. Die Blutabnahme zur Bestimmung der Serumtryptase (im Vergleich zur basalen Tryptase) 1-2 h nach einer Reaktion ist hilfreich zur Sicherung der Diagnose einer Anaphylaxie [16].

Spättypreaktionen können sich unter anderem als Exanthem unterschiedlichen Schweregrads manifestieren, aber auch mit der oben dargestellten Symptomatik. Bei Spättypreaktionen treten die Symptome mit zeitlicher Verzögerung von einigen Stunden auf und werden daher nicht während der Überwachungsphase in der Impfstelle erfasst.

\section{Therapie einer Anaphylaxie bedingt durch COVID-19- Impfstoffe}

Anaphylaktische Reaktionen erfordern die unmittelbare Behandlung, essenziell ist bei raschem Verlauf und Beteiligung mehrerer Organe die Gabe von Volumen und Adrenalin intramuskulär (• Tab. 2; $[16,17])$.

Wichtig ist auch die richtige Lagerung des Patienten (Schocklage). Blutdruck, Puls und Sauerstoffsättigung müssen adäquat überwacht werden, die Gabe von Sauerstoff wird empfohlen $[16,17]$.

Antihistaminika sind insbesondere bei Urtikaria wirksam, Kortikosteroide wirken unter anderem einem biphasischen Verlauf entgegen. Wegen dieser Möglichkeit eines biphasischen Verlaufs wird zumindest bei schwereren Reaktionen die Überwachung des Patienten über $24 \mathrm{~h}$ empfohlen [16].

Spättypreaktionen im Sinne von Exanthemen werden in Abhängigkeit vom Schweregrad bei mildem Verlauf mit topischen Kortikosteroiden sonst mit systemischen Kortikosteroiden behandelt. Gegen Juckreiz sind Antihistaminika begrenzt wirksam.

Um diese Therapie zu gewährleisten, wird eine Mindestausstattung an Pharmaka (•Tab. 2) und medizinischem Material ( Tab. 3) benötigt, die in jeder Impfstelle verfügbar sein müssen. $\mathrm{Zu}$ dem muss das Impfpersonal in der Erkennung und Behandlung schwerer allergischer Reaktionen geschult sein.

\section{Diagnostik}

Prick- und intrakutane Hauttests sowie die Analyse von Blutproben auf antigenspezifisches IgE, ggf. auch IgG und IgM und Provokationstests werden zur Identifizierung empfohlen, obwohl die Tests keinen $100 \%$ igen negativen Vorhersagewert haben $[19,20]$. Der Einsatz des Basophilenaktivierungstests (BAT) wurde beschrieben, ist jedoch bislang auf spezialisierte Zentren beschränkt und nicht Teil der Routinediagnostik [8].

\section{Diskussion}

Die klinischen Manifestationen der ersten Fälle in Großbritannien, den USA und Kanada passen zum klinischen Leitbild der Anaphylaxie: Sie traten innerhalb von Minuten nach den Injektionen auf, die Symptome waren typisch und alle sprachen auf Adrenalingabe an. Das Auftreten bei der ersten Exposition ist nicht typisch für IgE-vermittelte Reaktionen; allerdings könnte eine bereits bestehende Sensibilisierung auf eine Komponente des Impfstoffs diese Beobachtung erklären [4].

Die Berichte über diese Reaktionen in der Presse haben unter den Patienten mit Allergie massive Ängste bezüglich der Risiken der neuen Impfstoffe ausgelöst. Mit allergologischer Expertise betrachtet sind sie jedoch fast unvermeidlich, wenn eine Impfung für Millionen von Menschen begonnen wird.

Anaphylaxien aufImpfstoffe treten bei allen bislang bekannten Impfstoffen auf mit einer Häufigkeit von durchschnittlich etwa 1 auf 1.000.000 Injektionen [21]. Auch wenn diese Häufigkeit bei Applikation der beiden mRNA-Impfstoffe in den ersten Analysen ungefähr 10-mal häufiger war (1 auf 100.000 Injektionen), ist dies zunächst nichts Ungewöhnliches. Es unterstreicht die Notwendigkeit eines intensiven Monitorings sowie konstruktiver und proaktiver Handlungsempfehlungen, damit kausale Mechanismen definiert, Risikogruppen identifiziert und Strategien implementiert werden können, die die Behandlung und Prävention erleichtern [21].

Wichtig ist, dass eine Anaphylaxie jeden Geimpften überall und jederzeit tref- 
Tab. 2 Pharmakotherapie einer Anaphylaxie bei Kindern, Jugendlichen und Erwachsenen unter ambulanten Bedingungen. (Nach [16])

\begin{tabular}{|c|c|c|c|c|c|}
\hline \multirow[t]{2}{*}{ Wirkstoff } & \multirow{2}{*}{$\begin{array}{l}\text { Applikations- } \\
\text { weg }\end{array}$} & \multicolumn{4}{|c|}{ Körpergewicht } \\
\hline & & $<7,5$ kgKG & \multicolumn{3}{|c|}{$(7,5-30 \mathrm{kgKG})^{\mathrm{d}}$} \\
\hline Adrenalin & Intramuskulär & \multicolumn{4}{|l|}{$50-600 \mu \mathrm{g}$} \\
\hline Adrenalin & $\begin{array}{l}\text { Autoinjektor, } \\
\text { intramuskulär }\end{array}$ & $\begin{array}{l}\text { Nicht zu- } \\
\text { gelassen }\end{array}$ & $150 \mu \mathrm{g}$ & $300 \mu \mathrm{g}$ & $\begin{array}{l}\text { 1- bis } 2-\mathrm{mal} 300 \mu \mathrm{g} \\
\text { oder } 500 \mu \mathrm{g}\end{array}$ \\
\hline Adrenalin & $\begin{array}{l}\text { Inhalativ, Ver- } \\
\text { nebler }\end{array}$ & \multicolumn{4}{|l|}{$2 \mathrm{ml}^{\mathrm{b}}$} \\
\hline Adrenalin & Intravenös ${ }^{\mathrm{a}}$ & \multicolumn{4}{|c|}{ Titrierend, Boli $1 \mu \mathrm{g} / \mathrm{kgKG}$} \\
\hline Dimetinden & Intravenös & $1 \mathrm{ml}^{\mathrm{c}}$ & $\begin{array}{l}1 \mathrm{ml} / 10 \mathrm{kgKG}{ }^{c} \\
\text { (maximal } 4 \mathrm{ml} \text { ) }\end{array}$ & $\begin{array}{l}1 \text { Ampul- } \\
\text { le }=4 \mathrm{ml}^{\mathrm{c}}\end{array}$ & $\begin{array}{l}\text { 1-2 Ampul- } \\
\text { len }=4-8 \mathrm{ml}^{\mathrm{c}} \\
(1 \mathrm{ml} / 10 \mathrm{kgKG})\end{array}$ \\
\hline Prednisolon & Intravenös & $50 \mathrm{mg}$ & $100 \mathrm{mg}$ & $250 \mathrm{mg}$ & $500-1000 \mathrm{mg}$ \\
\hline $\begin{array}{l}\text { Salbutamol } \\
\text { Terbutalin }\end{array}$ & Inhalativ & $\begin{array}{l}2 \text { Hübe DA } \\
\text { per Spacer }\end{array}$ & $\begin{array}{l}2 \text { Hübe DA per } \\
\text { Spacer }\end{array}$ & $\begin{array}{l}\text { 2-4 Hübe DA } \\
\text { per Spacer }\end{array}$ & $\begin{array}{l}\text { 2-4 Hübe DA per } \\
\text { Spacer }\end{array}$ \\
\hline Volumen & $\begin{array}{l}\text { Bolus ( } \mathrm{NaCl} \\
0,9 \%)\end{array}$ & $20 \mathrm{ml} / \mathrm{kgKG}$ & $20 \mathrm{ml} / \mathrm{kgKG}$ & $\begin{array}{l}10-20 \mathrm{ml} / \\
\mathrm{kgKG}\end{array}$ & $10-20 \mathrm{ml} / \mathrm{kgKG}$ \\
\hline Sauerstoff & Inhalativ & $2-10 \mathrm{l} / \mathrm{min}$ & $5-121 / \min$ & $5-121 / \min$ & $5-121 / \min$ \\
\hline \multicolumn{6}{|c|}{ 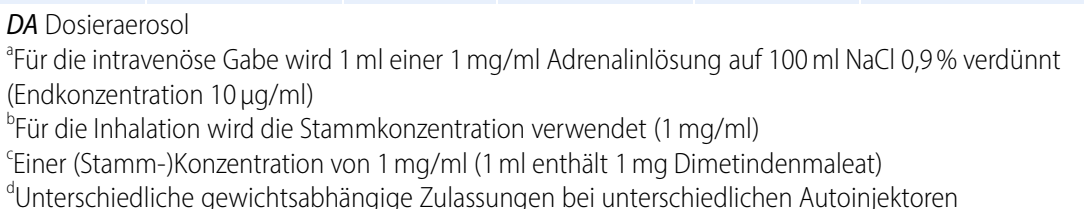 } \\
\hline
\end{tabular}

fen kann, auch unabhängig davon, ob bereits allergische Erkrankungen vorbekannt sind. Es gibt auch keine Korrelation der Inzidenz mit Alter oder Geschlecht $[16,20]$. Daher ist wichtig, dass jede Impfstelle und jeder Impfende darauf vorbereitet ist, schwere allergische Reaktionen zu erkennen und zu behandeln ( $\bullet$ Tab. 2 und 3 ).

\section{》) Eine Anaphylaxie kann jeden Geimpften überall und jederzeit treffen}

Die mRNA-Impfstoffe enthalten ein lipidbasiertes Nanopartikelträgersystem, das den schnellen enzymatischen Abbau der mRNA verhindert. Das lipidbasierte Nanopartikelträgersystem wird zusätzlich durch ein PEG-2000-Lipidkonjugat stabilisiert, das eine hydrophile Schicht bildet und die Halbwertszeit verlängert. Es gab zuvor keine zugelassenen mRNAImpfstoffe und auch keine anderen Impfstoffe, die auf dieser Trägertechnologie basieren. Daher bestehen keine früheren Erfahrungen über die Wahrscheinlichkeit oder den Mechanismus allergischer
Reaktionen im Zusammenhang mit mRNA-Impfstoffen.

In Kürze wird voraussichtlich ein SARS-CoV-2-Impfstoff der Firma AstraZeneca mit einem Adenovirusträger die Zulassung erhalten. Allergologisch interessant ist hier eine Proteinuntereinheit, die mit Polysorbat 80 formuliert wird, einem nichtionischen Tensid und Emulgator, der eine ähnliche Struktur wie PEG aufweist [21, 22]. In den USA werden in den nächsten Monaten wahrscheinlich mindestens fünf weitere neue Impfstoffe auf den Markt kommen, andere befinden sich in der Entwicklung [22]. Diese Entwicklung wird auch bei uns in der EU weitergehen. Es wird daher wichtig sein, das Vertrauen der Öffentlichkeit aufrechtzuerhalten mit wirksamen Strategien zum Monitoring und zur Verbesserung der Sicherheit auf individueller und auf Bevölkerungsebene [23-25].

In Zukunft könnte auf Grundlage der mRNA-Impfstoff-Plattform eine personalisierte Impfstoffentwicklung ermöglicht werden, mit der sich der sicherste und wirksamste Impfstoff auf individueller Ebene maßgeschneidert herstellen lässt [26].
Tab. 3 Materielle Ausstattung zur Be-

handlung anaphylaktischer Reaktionen in ambulanten Einrichtungen/Impfzentren. (Nach [16])

Stethoskop

Blutdruckmessgerät

Pulsoxymeter, evtl. Blutzuckermessgerät Stauschlauch, Venenverweilkanülen (in verschiedenen Größen), Spritzen, Infusionsbesteck, Pflaster zur Fixierung der Kanülen

Sauerstoff und Vernebler-Set mit Sauerstoffmaske (verschiedene Größen)

Beatmungsbeutel mit Masken (verschiedene Größen)

Absaugvorrichtung

Ggf. Guedel-Tubus

Volumen (z. B. balancierte Vollelektrolytlösung)

Arzneistoffe zur Injektion: Adrenalin, Glukokortikoid, H1-Rezeptor-Antagonist

Kurz wirksamer $\beta 2$-Adrenozeptor-Agonist, z. B. Salbutamol zur Inhalation (bevorzugt als Inhalationslösung zur Anwendung über Vernebler-Set mit Maske, ggf. alternativ als Dosieraerosol mit Inhalierhilfe/Spacer/ Maske, Autohaler o. Ä.)

Automatisierter externer Defibrillator

Auf immunologischer Ebene bleiben in Bezug auf COVID-19-Impfstoffe viele Fragen offen. Was genau sind die Korrelate der schützenden Immunität nach einer natürlichen Infektion oder Impfung und können diese ein Monitoring ermöglichen? Wie lange wird die Immunität anhalten? Welche Komponente des Impfstoffs ist für die allergischen Reaktionen verantwortlich und kann dies bei der Impfstoffentwicklung oder -weiterentwicklung berücksichtig werden?

Solange diese Fragen nicht beantwortet sind und generell ein Mangel an Impfstoff besteht, sollte mit den (guten!) vorhandenen Impfstoffen eine möglichst effiziente und sichere Impfstrategie verfolgt werden. Aus allergologischer Sicht sollten hierfür Impfzentren und andere Impfende die Vorbeugung, Diagnose und Behandlung schwerer allergischer Reaktionen berücksichtigen. Entsprechend sollten notwendige Medikamente und Ausrüstung in Impfzentren, aber auch in $\mathrm{Al}$ ters- und Pflegeheimen und für mobile Impfteams zur unmittelbaren Anwendung verfügbar sein. Die dargestellten potenziell allergenen/immunogenen Bestandteile sollten bei den definierten Risi- 
kopopulationen vor einer Impfung, aber auch bei Patienten nach entsprechenden Impfreaktionen getestet werden, um das verantwortliche Allergen zu identifizieren und um gegebenenfalls die notwendigen Maßnahmen für die zweite Impfdosis treffen zu können. Es ist wichtig zu betonen, dass derzeit keine zugelassenen Testallergene für diese Indikation verfügbar sind. Diese sollten baldmöglichst verfügbar gemacht werden, wozu verschiedene Entwicklungsprogramme angelaufen sind.

\section{Fazit für die Praxis}

- Kein Allergiker sollte ohne hinreichenden Grund von einer COVID-19Impfung ausgeschlossen werden.

- Der überwiegende Teil aller Allergiker kann eine COVID-19-Impfung mit den zugelassenen Impfstoffen erhalten.

- Allergiker, die Risikogruppen angehören, sollten vor einer COVID19-Impfung einer allergologischen Abklärung zugeführt werden.

- Patienten mit Allergien sollten nach einer Impfung für $\mathbf{3 0}$ min überwacht werden.

- Anaphylaktische Reaktionen können bei vorbekannten Allergien, jedoch auch ohne vorherige Hinweise auftreten. Jeder Impfarzt muss daher auf Anaphylaxiebehandlungen vorbereitet sein und über entsprechende Sachkenntnis verfügen.

- Impfarzt und -personal müssen in der Erkennung und Behandlung schwerer allergischer Reaktionen geschult sein.

- Eine Mindestausstattung an Medikamenten und Instrumentarium muss in jeder Impfstelle zur unmittelbaren Anwendung verfügbar sein. Das gilt für Impfzentren, Arztpraxen, mobile Impfteams in Pflegeheimen, Patientenwohnungen etc.

- Bei (vermeintlichen) allergischen Reaktionen auf die Impfstoffe sollte eine allergologische Abklärung in einem spezialisierten Zentrum erfolgen.

\section{Korrespondenzadresse}

Prof. Dr. med. L. Klimek

Zentrum für Rhinologie und Allergologie

An den Quellen 10, 65183 Wiesbaden,

Deutschland

ludger.klimek@allergiezentrum.org

\section{Einhaltung ethischer Richtlinien}

Interessenkonflikt. L. Klimek, J. Eckrich, J. Hagemann, I. Casper und J. Huppertz geben an, dass kein Interessenkonflikt besteht.

Für diesen Beitrag wurden von den Autoren keine Studien an Menschen oder Tieren durchgeführt. Für die aufgeführten Studien gelten die jeweils dort angegebenen ethischen Richtlinien.

\section{Literatur}

1. Polack FP, Thomas SJ, Kitchin N, Absalon J, Gurtman A, Lockhart S et al (2020) Safety and efficacy of the BNT162b2 mRNA Covid-19 vaccine. N Engl J Med. https://doi.org/10.1056/ NEJMoa2034577

2. Jackson LA, Anderson EJ, Rouphael NG, RobertsPC Makhene M, Coler RN et al (2020) An mRNA vaccine against SARS-CoV-2 - preliminary report. $\mathrm{N}$ Engl J Med 383(20):1920-1931

3. Klimek LNN, Cabanillas B, Jutel M, Bousquet J, Akdis CA (2021) Potential allergenic components of the mRNA-1273 vaccine for COVID-19: possible roles for polyethlene glycol and IgG-mediated complement activation. Allergy (in press)

4. Klimek LNN, Hamelmann E et al (2020) Severe allergic reactions after COVID-19-Vaccination with the Pfizer/BioNTech Vaccine in Great Britain and USA Position Statement of the German allergological Societies AeDA, DGAKI and GPA. Allergo J Int. https://doi.org/10.1007/s40629-02000160-4 (in preparation)

5. Dooling K, McClung N, Chamberland M, Marin M Wallace M, Bell BP et al (2020) The advisory committee on immunization practices' interim recommendation for allocating initial supplies of COVID-19 vaccine-United States, 2020. MMWR Morb Mortal Wkly Rep 69(49):1857-1859

6. Inglut CT, Sorrin AJ, Kuruppu T, Vig S, Cicalo J, Ahmad $\mathrm{H}$ et al (2020) Immunological and toxicological considerations for the design of liposomes. Nanomaterials. https://doi.org/10. 3390/nano10020190

7. Allen TM, Hansen C, Martin F, Redemann C, YauYoung A (1991) Liposomes containing synthetic lipid derivatives of poly(ethylene glycol) show prolonged circulation half-lives in vivo. Biochim Biophys Acta 1066(1):29-36

8. Klimek LJM, Akdis C (2020) ARIA-EAACI statement on severe allergic reactions to COVID-19 vaccines - an EAACI-ARIA Position Paper. Allergy. https://doi.org/10.1111/all.14726

9. Sellaturay P, Nasser S, Ewan P (2020) Polyethylene glycol-induced systemic allergic reactions (anaphylaxis). J Allergy Clin Immunol Pract. https://doi. org/10.1016/j.jaip.2020.09.029

10. Stone CA, Liu Y, Relling MV, Krantz MS, Pratt AL, Abreo A et al (2018) Immediate hypersensitivity to polyethylene glycols and polysorbates: more common than we have recognized. J Allergy Clin Immunol Pract 7(5):1533-1540.e8

11. Wenande E, Garvey LH (2016) Immediate-type hypersensitivity to polyethylene glycols: a review. Clin Exp Allergy 46(7):907-922

12. Wylon K, Dölle S, Worm M (2016) Polyethylene glycol as a cause of anaphylaxis. Allergy Asthma Clin Immunol 12(1):67

13. Krantz MLY, Phillips EJ, Stone CA (2020) COVID-19 vaccine anaphylaxis: PEG or not? Allergy (in press)

14. Krantz MS, Liu Y, Phillips EJ, Stone CA Jr. (2020) Anaphylaxis to PEGylated liposomal echocardiogram contrast in a patient with lgEmediated macrogol allergy. J Allergy Clin Immunol Pract 8(4):1416-1419e3

15. Szebeni J (2014) Complement activation-related pseudoallergy: a stress reaction in blood triggered by nanomedicines and biologicals. Mol Immunol 61:163-173

16. Ring J, Beyer K, Biedermann T, Bircher A, Fischer M Fuchs Tetal (2021) Guidelines (S2) to acute therapy and management of anaphylaxis — update 2021. Allergo JInt (in press)

17. Klimek L, Worm M, Lange L, Beyer K, Rietschel E, Vogelberg $C$ et al (2020) Management von Anaphylaxiegefährdeten Patienten während der Covid-19-Pandemie. Allergo J 29(7):16-26

18. CDC (2021) COVID-19 Response Team, FDA-Allergic Reactions Including Anaphylaxis After Receipt of the First Dose of PfizerBioNTech COVID-19 Vaccine-United States, December 14-23, 2020: Centers for Disease Control and Prevention. https://www.cdc.gov/mmwr/ volumes/70/wr/mm7002e1.htm. Zugegriffen: 6. Jan. 2021 (updated 06.01.2021)

19. Castells M (2017) Diagnosis and management of anaphylaxis in precision medicine. J Allergy Clin Immunol 140(2):321-333

20. Klimek L, Bergmann KC, Brehler R, Pfützner W Worm M, Hartmann K et al (2021) Allergologische Diagnostik und Therapien bei COVID-19 Impfungen: Praktische Handlungsempfehlungen. Empfehlungen von AeDA, DGAKI und GPA. Allergo J (in press)

21. Stone CA Jr., Rukasin CRF, Beachkofsky TM, Phillips EJ (2019) Immune-mediated adverse reactions to vaccines. Br J Clin Pharmacol 85(12):2694-2706

22. Krammer $F$ (2020) SARS-CoV-2 vaccines in development. Nature 586(7830):516-527

23. Jakob T, Klimek L (2020) Allergologie in Zeiten von Covid-19. Allergo J 29(3):3

24. KlimekL, Pfaar O, Worm M, Bergmann K-C, BieberT, Buhl $R$ et al (2020) Allergen-Immuntherapie in der aktuellen Covid-19-Pandemie a, b, c. Allergo J 29(3):17-25

25. Klimek L, Pfaar O, Worm M (2020) Anwendung von Biologika bei allergischen und Typ-2-entzündlichen Erkrankungen in der aktuellen COVID-19Pandemie - ein Positionspapier von AeDA, DGAKI, GPA, ÖGAI, LGAI, ÖGP, ARIA und EAACI. Use of biologicals in allergic and type-2 inflammatory diseases in times of the current COVID-19 pandemic - Position paper of AeDA, DGAKI, GPA, ÖGAI, LGAI, ÖGP, ARIA und EAACI. Allergologie 43(7):255

26. Poland GA, Ovsyannikova IG, Kennedy RB (2018) Personalized vaccinology: a review. Vaccine 36(36):5350-5357 
Hier steht eine Anzeige.

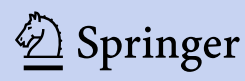

